\title{
Response to the letter to the editor entitled "maxillary sinus surgery in maxillary sinusitis with cystic fibrosis" of Alper Nabi Erkan, MD, Assoc. Prof
}

\author{
Domenic Vital · David Holzmann
}

Published online: 27 February 2013

(c) Springer-Verlag Berlin Heidelberg 2013

\section{Sir,}

It is in fact true that a wide opening of the sinuses should be accomplished in patients with cystic fibrosis-related chronic rhinosinusitis. In particular, this is of note for the maxillary sinus as there the greatest amount of mucus retention is encountered and we thus advocate an extensive sinusotomy. To do so, we open the maxillary sinus by removing greater parts of the medial maxillary wall, which correlates to a certain extent with the objection of Dr. Erkan. However, due to rapid progress in transnasal endoscopic surgery and along studies on long-term follow-up, there is meanwhile broad consensus that the window in the inferior meatus does not play an important role as long as it is not connected with the enlarged natural ostium of the maxillary sinus (i.e. in the middle meatus). Thick mucous retention harbouring specific bacteria like $P$. aeruginosa or $S$. aureus activates inflammatory processes and leads to nasal polyp growth. Thereafter, the maxillary sinus neither will be drained nor can be rinsed with any irrigation. In the "end stage", the entire maxillary sinus is filled with this enormously tenacious secretion and not only in the inferior portion. 